\title{
Persée
}

\author{
http://www.persee.fr
}

\section{Le culte de la persuasion. Peithô en Grèce ancienne}

\author{
Vinciane Pirenne-Delforge \\ Revue de l'histoire des religions, Année 1991, Volume 208, Numéro 4 \\ p. $395-413$
}

Voir l'article en ligne

La persuasion trouve un champ d'action privilégié dans la pratique de la politique et l'exercice de la justice. Elle relève alors d'un art, d'une technique. Mais Peithô est également une déesse à laquelle certaines cités rendent un culte. L'étude de ces manifestations religieuses, en regard de l'exploitation littéraire du thème de la persuasion, met en évidence les interférences du politique et de l'érotique, ainsi que l'importance de l'institution matrimoniale dans l'affirmation de la « civilisation » de la polis.

\section{Avertissement}

L'éditeur du site "PERSEE » - le Ministère de la jeunesse, de l'éducation nationale et de la recherche, Direction de l'enseignement supérieur, Sous-direction des bibliothèques et de la documentation - détient la propriété intellectuelle et les droits d'exploitation. A ce titre il est titulaire des droits d'auteur et du droit sui generis du producteur de bases de données sur ce site conformément à la loi n`98-536 du 1er juillet 1998 relative aux bases de données.

Les oeuvres reproduites sur le site « PERSEE » sont protégées par les dispositions générales du Code de la propriété intellectuelle.

Droits et devoirs des utilisateurs

Pour un usage strictement privé, la simple reproduction du contenu de ce site est libre.

Pour un usage scientifique ou pédagogique, à des fins de recherches, d'enseignement ou de communication excluant toute exploitation commerciale, la reproduction et la communication au public du contenu de ce site sont autorisées, sous réserve que celles-ci servent d'illustration, ne soient pas substantielles et ne soient pas expressément limitées (plans ou photographies). La mention Le Ministère de la jeunesse, de l'éducation nationale et de la recherche, Direction de l'enseignement supérieur, Sous-direction des bibliothèques et de la documentation sur chaque reproduction tirée du site est obligatoire ainsi que le nom de la revue et- lorsqu'ils sont indiqués - le nom de l'auteur et la référence du document reproduit.

Toute autre reproduction ou communication au public, intégrale ou substantielle du contenu de ce site, par quelque procédé que ce soit, de l'éditeur original de l'oeuvre, de l'auteur et de ses ayants droit.

La reproduction et l'exploitation des photographies et des plans, y compris à des fins commerciales, doivent être autorisés par l'éditeur du site, Le Ministère de la jeunesse, de l'éducation nationale et de la recherche, Direction de l'enseignement supérieur, Sous-direction des bibliothèques et de la documentation (voir http://www.sup.adc.education.fr/bib/). La source et les crédits devront toujours être mentionnés. 
VINCIANE PIRENNE-DELFORGE

(Aspirant da F.N.R.S.)

Université de Lì̀ge

\section{LE CULTE DE LA PERSUASION PEITHÔ EN GRÈCE ANCIENNE}

La persuasion trouve un champ d'action privilégié dans la pratique de la politique et l'exercice de la justice. Elle relève alors d'un art, d'une technique. Mais Peithó est également une déesse à laquelle certaines cilés rendent un culte. L'étude de ces manifestations religieuses, en regard de l'exploitation littéraire du thème de la persuasion, met en évidence les interférences du politique et de l'érotique, ainsi que l'importance de l'institution matrimoniale dans l'affirmation de la "civilisation" de la polis.

The Cult of the persuasion. Peitho in Ancient Greece.

Persuasion essentially depends on politics and justice. Then it is an art, a technique. But Peithô is also a goddess worshipped in some city-states. The study of all these religious manifestations, together with the literature which uses the theme of the persuasion, shows how politics and erotic are closely connected, and how important is the matrimonial institution in Greek polis' civilization. 
Peitho is a continuum within which divine and secular, erotic and non-erotic come together.

R. G. A. Buxton,

Persuasion in Greek Tragedy, p. 31 .

Persuader, c'est amener quelqu'un à croire, à penser, à vouloir, à faire quelque chose, par une adhésion complète, sentimentale autant qu'intellectuelle ${ }^{1}$. Pour les Grecs de l'Antiquité, peitho, la persuasion, quand elle est action, relève d'un art, d'une technique, mais elle fait également partie du cortège des divinités.

Explicitement définie dans le Gorgias de Platon comme " ouvrière de persuasion $~^{2}$, la rhétorique serait apparue en Sicile, selon la tradition, après la chute des tyrans de Syracuse dans le deuxième quart du ve siècle avant notre ère ${ }^{\mathbf{3}}$. Le cadre de son émergence serait soit la mise en place de discours délibératifs liés à une forme primitive de démocratie, soit l'élaboration de discours judiciaires pour le règlement de litiges liés au changement de régime. Politique et justice, tels sont donc, dès l'origine, les deux champs d'action de la persuasion quand elle devient l'objet d'une technique*.

La peitho, même dans les acceptions que nous appellerons " laïques " pour faire bref, ne se départira jamais complètement de l'aura divine qui l'accompagnait dès ses premiers pas, mais c'est en tant que divinité à part entière que nous l'étudierons aujourd'hui, car elle recevait bien un culte. Or, quand on ne l'oblitère pas complètement, cet aspect parti-

1. Robert, Dictionnaire français, 1989 , s.v.

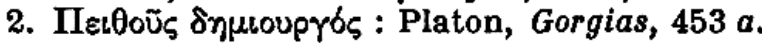

3. G. G. Kennedy, The Art of Persuasion in Greece, Princeton, 1963, p. 58-61, spéc. p. 58 , n. 5 .

4. Cf. Marie-Pierre Noël, La persuasion et le sacré chez Gorgias, in $B A G B$ (1989), p. 139-151, spéc. 139. - Signalons en passant que, sur le plan politique, sophistique et rhétorique sont inséparables et développent des techniques solidaires dont le but ultime, dans les deux cas, est la persuasion : M. Détienne, Les mattres de vérité dans la Grèce archatque, Paris, 1967, p. 119-124. 
culier ne vient jamais que se greffer, un peu artificiellement, sur des études élaborées dans un autre but ${ }^{5}$. Et quand la déesse a les honneurs de l'analyse, les interprétations laissent à désirer en ce qu'elles ne tiennent pas compte de l'arrièreplan culturel sur lequel vient s'inscrire la peitho ${ }^{6}$. Il n'est donc pas inutile d'esquisser, même rapidement, le visage de la persuasion quand elle entre en littérature, avant d'interpréter son rôle dans la vie cultuelle des cités où on la rencontre.

Une première remarque s'impose : la persuasion n'emprunte pas nécessairement le chemin des mots, même si le langage est une de ses principales voies d'action. « Les cadeaux convainquent même les dieux et l'or est, parmi les mortels, plus puissant qu'un millier de mots " dira la Médée d'Euripide (v. 964-965) ${ }^{7}$; de même Nestor, exhortant Agamemnon au chant IX de l'Iliade : "Allons ! examinons, s'il en est temps encore, les moyens de persuader ( $\left.\pi \varepsilon \pi i \theta_{\circ} \circ \mu \varepsilon v\right)$ et d'apaiser Achille par de beaux présents et de douces paroles " (v. 112$113)^{8}$. Quant à Empédocle, il qualifiera la vue et le toucher de moyens « par lesquels la grand'route de la persuasion s'enfonce dans l'esprit des hommes ${ }^{9}$.

C'est avec Hésiode que la persuasion emprunte le plus clairement le statut de divinité. Dans la Théogonie (v. 349), elle est fille d'Okéanos et de Téthys, sœur de Mètis, de Tychè et des milliers d'autres Océanides ${ }^{10}$. Dans les Travaux et les

5. Par ex. R. G. A. Buxton, Persuasion in Greek Tragedy, Cambridge, 1982, p. 31-48 ; N. P. Gross, Amatory Persuasion in Antiquity, London, 1985, p. 15-31.

6. Les principaux travaux sont: $\mathrm{O}$. Jahn, Peitho, die Götin des Überredung, Greifswald, 1846 ; F. Voigt, art. Peitho, in $R E$, XIX, 1 (1937), col. 1795-1813; P. Weizsäcker, art. Peitho, in Roscher, Lexikon..., III, 2 (1897-1909), col. 17951813. Voir aussi F. W. Hamdorf, Griechische Kultpersonniflkationen, Mainz, 1964 , p. $63-65,117-118$; A. Shapiro, The Origins of Allegory in Greek Art, in Boreas, 9 (1986), p. 4-23.

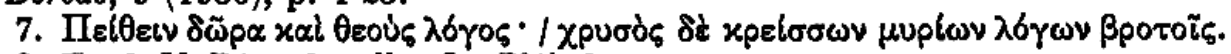

8. Trad. V. Bérard, coll. "La Pléiade *.

9. Empédocle, 31 B 133 Diels-Kranz ${ }^{6}$ (= Clément d'Alexandrie, Stromates, V, 82) : ... है b甲

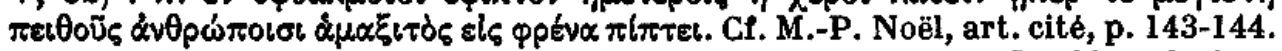

10. Sur la signification de cette filiation, cf. J. Rudhardt, Le theme de l'eau primordiale dans la mythologie grecque, Berne, 1971 (Travaux publiess sous les auspices de la Société suisse des Sciences humaines, 12), p. 31, 69-70. On lira aussi les intéressantes remarques de M. L. West, Hesiod Theogony, Oxford, 1966, p. 260-269. 
jours, elle se substitue, en compagnie des Heures et des Charites ${ }^{11}$, à la déesse Aphrodite pour parer Pandore, la première femme, de tous les attributs de la séduction féminine (v. 73-74) ${ }^{12}$. Mais les mots ne constituent pas le passage obligé de la Persuasion quand elle devient amoureuse, car c'est Hermès qui est appelé en renfort pour mettre au cœur de Pandore des mots trompeurs (v. 76-77) et lui donner l'usage de la parole (v. 79) ${ }^{13}$. Colliers d'or et guirlandes de fleurs sont les dons des compagnes d'Aphrodite, ce qui ne laisse pas d'évoquer le pouvoir enjôleur de ces enjolivements ${ }^{14}$. Et Peitho de s'identifier à la séduction quand elle passe dans la sphère amoureuse $^{15}$; elle devient alors le nom générique de la stratégie qui suscite le désir, sans qu'il soit toujours possible de faire le départ entre la déesse et la " notion abstraite "16. Un exemple : dans les Trachiniennes, Sophocle exprime au travers du chœur l'espoir de Déjanire de voir revenir Héraclès à nouveau amoureux après s'être revêtu de la tunique magique (v. 660-662). Or le funeste liquide cédé par Nessos devient dans l'espérance de la malheureuse une onction de persua-

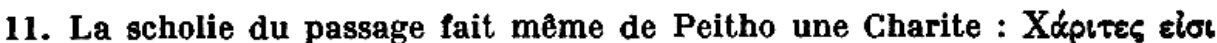

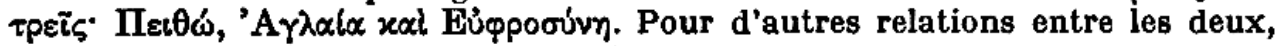
cf. P. Weizsăcker, art. cité, col. 1796.

12. Cf. M. L. West, Hesiod Works and Days, Oxford, 1978, p. 161-162.

13. Cf. M. L. West, $W \& D$, op. cit., p. 163-164; J. Kambitsis, "O $\mathrm{O}_{\mu \alpha \tau \tau \alpha}$

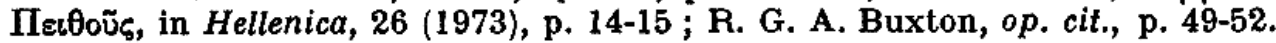
- Marcel Détienne (op. cit., p. 62) relie apparemment le pouvoir de Peitho à la seule action de la parole : $\propto$... Peitho, qui est sans hésitation la puissance de la parole telle qu'elle s'exerce sur autrui, sa magie, sa séduction, telle qu'autrui la subit *. - Sur Hermès, cf. Laurence Kahn, Hermès passe ou les ambigultés de la communication, Paris, 1979, p. 125-127, 141.

14. La " parade amoureuse " d'Aphrodite dans l'Hymne homérique en son honneur intègre également des boucles, des spirales courbes, des ornements en forme de fleur, des colliers (v. 163). Pour une illustration de la parenté des thèmes de l'or et de la séduction, cf. P. Brulé, La fille d'Athènes. La religion des flles a Athènes d l'époque classique. Mythes, cultes et sociêté, Paris, 1987 (Annales litt. de l'Univ. de Besançon, 363. Centre de Recherches d'Histoire Ancienne, 76), p. 267.

15. N. P. Gross, op. cit., passim. - Peitho devient fille d'Aphrodite chez Sappho (frag. 200 Lobel-Page) et dans les Suppliantes d'Eschyle (v. 1039-1041). Cf. aussi Ibycus, frag. 288 Page ; Pindare, Pyth., IV, 219 ; IX, $39 a$.

16. R. G. A. Buxton, op. cit., p. 30, qui parle des Suppliantes d'Eschyle (v. 523), souligne très justement que, pour l'auteur et son auditoire, ce genre de distinction n'avait pas le même poids que pour un éditeur moderne qui doit choisir entre majuscule et minuscule à l'initiale ! 
sion ! Point de parole dans ce cas, et un choix impossible à faire entre la divinité et son action.

La séduction et la force quasi-magique de peitho ${ }^{17}$ ne disparaitront pas complètement dès lors que la persuasion entrera dans le jeu du politique, mais ses moyens relèveront désormais d'une technique ${ }^{18}$.

Alcman offre la première mention qui nous soit conservée d'une relation entre la Peitho mythique et le domaine politique par le biais d'une généalogie originale : Persuasion est fille de Prométheia, sœur d'Eunomia et de Tychè ${ }^{19}$. Elle entre dès lors dans l'orbe du respect des lois et de la prévoyance en relation avec le destin ${ }^{20}$. La peitho ainsi comprise élargit à la communauté le mode des relations vécues par les individus et représente la persuasion tant érotique que politique.

En dépit de l'affirmation d'Euripide qu' " il n'est pas d'autre sanctuaire de Peitho que le logos et (que) son autel est dans le cœur des hommes $~^{21}$, il existe bel et bien des cultes de Peitho que nous allons à présent passer en revue.

\section{ATHÈNES}

Pausanias a conservé le souvenir d'un sanctuaire logé sur le flanc sud-ouest de l'Acropole d'Athènes et abritant des

17. Sur cet aspect magique, cf. M. Détienne, op. cit., p. 62-64; M.-P. Noël, art. cité, p. 145-151. - Mentionnons encore l'épisode de la tromperie de Zeus par Héra au chant XIV de l'Iliade : Héra sollicite l'assistance du ceste magique d'Aphrodite afin, soi-disant, de persuader Okéanos et Téthys de s'aimer à nouveau. De plus, une tradition fera de Peitho unie a Pan la mére d'Iynx, l'oiseau magique de l'amour : Souda, s.v. tưk; schol. Pindare, Ném., IV, 56. Cf. P. Weizsăcker, art. cité, col. 1806.

18. M.-P. Noël (art. cité, passim) a bien montré le rôle de Gorgias dans cette évolution.

19. Alcman, frag. 105 Calame (= Plut., Mor., 318 a).

20. Pour un commentaire du fragment, cf. R. G. A. Buxton, op. cit., p. 41-42.

21. Euripide, Antigone, frag. 170 Nauck $^{2}$ - A pareille époque surgit une polémique similaire touchant à la nature divine d'Eros, cette question devenant l'objet d'exercices oratoires; cf. Euripide, frag. 269 Nauck $^{2}$, et les réflexions de F. Lasserre, La figure d'Eros dans la poésie grecque, Lausanne, 1946, p. 116126, et mon article Eros en Gréce : dieu ou démon ?, in Anges et démons. Actes du Colloque de Liège et de Louvain-la-Neuve (25-26 novembre 1987), Louvain-la-Neuve, 1989 (* Homo Religiosus *, 14), p. 230. 
statues d'Aphrodite et de Peitho dont les originaux n'étaient plus visibles à son époque ${ }^{22}$. Aphrodite porte à cet endroit l'épithète de Pandémos qui la qualifie incontestablement en tant que divinité protectrice du démos, déesse à vocation politique donc ${ }^{23}$. Le nom des déesses en ce lieu est attaché à celui de Thésée qui aurait instauré leur culte en action de grâce après le synécisme de l'Attique ${ }^{24}$. Aphrodite et Peitho incarnent dès lors des vertus de concorde civile et d'harmonie politique projetées dans le passé reculé de la cité. Une autre tradition, qui attribue à Solon la fondation du culte d'Aphrodite Pandémos ${ }^{25}$, ramène à l'époque historique l'origine d'une dévotion que la vraisemblance, à défaut de tout autre critère, n'interdit pas de qualifier d'archaïque. Il semble en effet que le culte était suffisamment ancien pour rendre plausible aux yeux des Athéniens son association avec les noms parmi les plus prestigieux du passé politique de leur cité. Peut-être les réformes de Clisthène ont-elles également connu, à la fin du vi $^{\mathrm{e}}$ siècle, une référence à ces mêmes divinités que l'on retrouve, semble-t-il, à cette époque, sur une monnaie

22. Paus., I, 22, 3. - On a trouvé, parmi des inscriptions dédiées à Aphrodite dans un petit sanctuaire de la déesse signale par Pausanias $(I, 37,7)$ et situé près de Daphni, sur la voie menant à Eleusis, une dédicace à Peitho ( $I G, \mathrm{II}^{2}, 4583$ ), preuve supplémentaire de l'association des deux divinités dont, dans ce cas, le Périégète ne mentionne cependant pas des cultes associés.

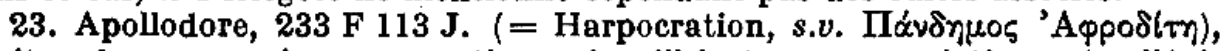
se situe dans une même perspective puisqu'il instaure une relation entre l'épithète et les réunions du démos sur l'agora à haute époque, même si son témoignage semble en contradiction avec celui de l'archéologie concernant la localisation de l'ancienne agora. Cf. mon article Epithètes cultuelles et interprétation philosophique. A propos d'Aphrodite Ourania et Pandémos à Athènes, in $A C$, 57 (1988), p. 142-157.

24. Cf. M. Moggi, I sinecismi interstatali greci, I, Pise, 1976, p. 44-81. - Sur l'appropriation de Thésée par les Athéniens et les hauts faits qui lui ont été attribués, cf. H. Herter, Theseus der Athener, in $R h M, 88$ (1939), p. 244-326. Plutarque (Thésée, 24, 1), parlant du synécisme, décrit Thésée allant de dème en dème, de famille en famille, pour les convaincre $(\varepsilon \tilde{\pi} \pi \varepsilon \theta \varepsilon)$ du bien-fondé de cette mesure.

25. Nicandre de Colophon, cité par Athénée, XIII, $569 d$-e et par Harpo-

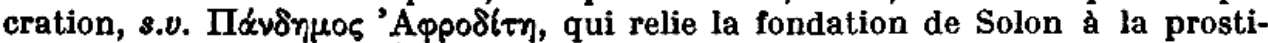
tution : cf. Epithètes cultuelles... art. cité, p. 145, 153-154. - Il n'est pas, dans ce texte, fait mention de Peitho, mais son association avec Aphrodite dans un tel contexte érotique est évidente : cf., par ex. a Corinthe, Pindare, frag. 122 Snell-Maehler. Cf. aussi J. H. Olivier, Demokratia, the Gods and the Free World, Baltimore, 1960, p. 106-117. 
qui porte d'un côté la tête d'Athéna et de l'autre deux têtes féminines bifrons ${ }^{26}$. Si cette dernière hypothèse est exacte, Aphrodite et Peitho étaient associées - dans une mesure évidemment difficile à évaluer - aux différentes étapes du parcours politique qui devait mener Athènes vers la démocratie. Sur le plan du rite, on sait que la cité offrait un sacrifice annuel à la divine Persuasion, du moins du temps d' Isocrate ${ }^{27}$. Mais on ne doit pas exclure l'éventuelle existence d'un autre sanctuaire où Peitho aurait été honorée seule et dont on n'aurait pas conservé de traces.

Si le rôle de la Peitho athénienne relève manifestement du politique, les fonctions conjointes des deux déesses au sein de la cité étaient peut-être également d'une autre nature, car il ne faut pas perdre de vue qu'Aphrodite est, en maints endroits d'Athènes, une déesse de la fécondité et de la fertilité ${ }^{28}$. Or la seule tradition conservée qui relie la Pandémos au nom de Solon émane d'une comédie qui en fait la patronne des courtisanes. J'ai déjà eu l'occasion de montrer ce que cette interprétation devait à une réécriture platonicienne des réalités cultuelles. Il faut cependant souligner que l'argument de vraisemblance, qui commande la lecture d'une pièce jouée devant un public averti, impose la prise en considération du rôle assumé par les deux déesses dans le rapprochement des sexes, qu'il soit vénal ou conjugal. D'autant que Plutarque a conservé, attaché au nom de Solon, le souvenir de quelques lois qui touchent à l'harmonie entre conjoints ${ }^{29}$.

26. Erika Simon, Aphrodite Pandemos auf attischen Münzen, in $S N R$, 49 (1970), p. 5-24, pl. 2, fig. 4. - R. G. A. Buxton (op. cit., p. 33-34) souligne le caractère hautement spéculatif de cette interprétation. Il nous semble cependant que le faisceau de convergences dégagé par $E$. Simon mérite d'être pris en consideration, fút-ce avec toute la prudence requise.

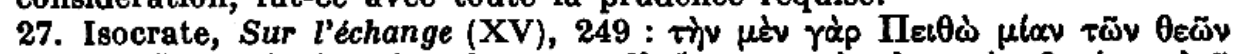

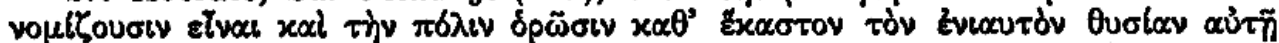
Tolouféviv... - Démosthène (Promium, 54) nous a également conservé le

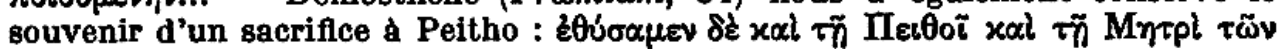

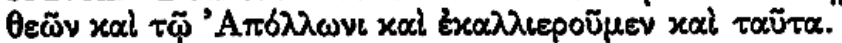

28. Cf. mon article Aspects orientaux du culte d'Aphrodite a Athènes, in Studia Phonicia $V$ : Phonicia and the East Mediterranean in the First Millennitu BC (Leuven, 14th-15th Nov. 1985), Leuven, 1987, passim et p. 153.

29. Plut., Mor., $138 d$ et Solon, 20: il s'agit principalement d'éviter les unions commandées par l'intérêt, et où charis et philotès n'auraient pas leur place. 
Il est probable que la comédie aura caricaturé de telles mesures en faisant du législateur lle créateur d'une prostitution permettant de juguler les ardeurs des jeunes gens!

Les préoccupations politiques de Peitho à Athènes nous sont encore confirmées par les Euménides d'Eschyle, tragédie dont la couleur locale ne fait aucun doute. Les Erinyes qui poursuivent Oreste de leur fureur vengeresse après le meurtre de Clytemnestre arrivent dans la patrie d'Athéna qu'elles menacent de stérilité et de mort après le verdict de l'Aréopage en faveur du matricide (v. 752-793). Et Athéna aura fort à faire pour les apaiser. S'adressant au chœur des antiques déesses, l'Olympienne invoque le saint respect de Peitho qui confère à ses paroles leur magique douceur (v. 885886), tandis que la conclusion de l'accord entre les divinités confirme la puissance de Persuasion dont Athéna bénit l'intervention (v. 969-971). C'est donc Peitho qui permet la transformation des Erinyes en Euménides, des Furies en Bienveillantes déesses, le passage de la vengeance familiale à la justice civile. Mais lorsque l'on s'aperçoit que les vierges farouches du début de la pièce finissent par prier les Moires d'accorder " aux vierges aimables de vivre aux côtés d'un époux " (v. 959-961), il semble légitime de faire l'hypothèse que l'harmonie sociale, dans laquelle s'inscrit l'institution matrimoniale, fait également partie des prérogatives de la Persuasion $^{30}$ alliée à Aphrodite dans la cité d'Athéna. On connait en ce sens le célèbre passage de Plutarque qui évoque les cinq divinités entourant les nouveaux époux : Zeus Teleios,

30. Très bien mis en évidence par J. Kambitsis, art. cité, p. 16-17. Cf. aussi R. G. A. Buxton, op. cit., p. 110-112. - Le mariage antique est avant tout une institution sociale dans la consécration de laquelle la religion officielle n'intervient que peu. L'évolution qui doit avoir eu lieu ne nous est guère accessible étant donné la nature de nos sources (cf. C. Vatin, Recherches sur le mariage et la condition de la femme mariée d l'époque hellénistique, Paris, 1970 [BEFAR, 216], p. 207-228). Si le patronage d'un certain nombre de divinités est bien connu, la démarche religieuse, dans ce cas, est d'ordre privé ; il n'existe pas, avant le I $^{\text {or }}$ siècle av. J.-C., de a mariage religieux . 
Héra Teleia, Aphrodite, Peitho et Artémis ${ }^{31}$, et le conseil qu'il donne aux fiancés d'invoquer Aphrodite, les Muses, Hermès, Peitho et les Charites ${ }^{\mathbf{2}}$.

\section{MÉGARE}

Pausanias visitant Mégare rencontre sur l'agora un temple d'Aphrodite dont l'épithète est Praxis et dont la statue en

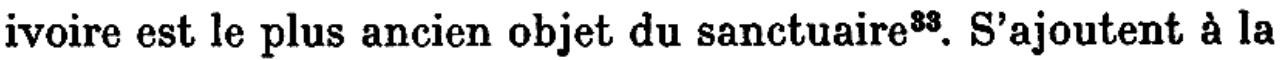
déesse, des statues d'Eros, Pothos et Himéros, œuvres de Scopas, tandis que Praxitèle a réalisé celles de Peitho et d'une autre déesse appelée Parègoros, que l'on pourrait traduire par "Parole encourageante ", ou " consolatrice ", le cas échéant. Devant un tel noyau cultuel, on a l'impression de lire le passage d'Hésiode relatif à la naissance d'Aphrodite ou encore l'épisode de la tromperie de Zeus par Héra dans

31. Plut., Mor., $264 b$ : il s'agit de la réponse à la question de savoir pourquoi, pendant les cérémonies du mariage, on allume cinq flambeaux. Cette question fait partie des Questions romaines, mais on peut supposer que Plutarque a ici procédé par analogie.

32. Plut., Mor., $138 c-d$. - On sait, grâce à quelques lambeaux d'informa-

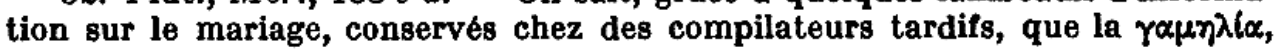
sacrifice lié au mariage, était offerte à Héra, à Aphrodite et aux Graces : Etym. Magnum, s.v. $\gamma \propto \mu \eta \lambda \uparrow \propto \alpha(220,50-57)$ : c'est le seul texte qui parle des divinités concernées, mais en considérant le sacrifice comme destiné aux éphèbes inscrits

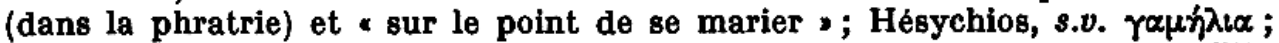
Pollux, III, 42. Certains textes semblent sous-entendre un sacrifice de nubilité (Pollux, VIII, 107 ; Harpocration, s.v. $\gamma \alpha \mu \eta \lambda \ell \alpha$, qui cite Didyme le Grammairien). Les Modernes ont répercuté l'alternative : V. Magnien (Le mariage chez les Grecs, in $A I P h O, 4$ [1936], p. 307-309 = Melanges F. Cumont) accepte l'hypothèse de deux sacriflces différents, le premier intervenant lors de l'inscription dans la phratrie, l'autre au moment de l'introduction de l'épouse; L. Deubner (Attische Festen, Hildesheim, $1969^{2}$ [1932], p. 232-234) se prononce en faveur d'un seul sacrifice, lors de l'introduction de la jeune épouse dans la phratrie de son mari; K. Latte (art. Phratrie, in $R E, X X, 1$ [1941], col. 752) et J. Labarbe (L'áge correspondant au sacrifice du kotreion et les données historiques du sixième discours d'Isée, in $B A B, 39$ [1953], p. 365) adoptent également cette position. P. Brulé (op. cit., p. 402-403) a dernièrement reposé le problème dans la perspective plus générale de la " religion des filles à Athènes ": tout en admettant la confusion des textes, il penche pour la diversité des démarches que recouvre le nom $\gamma \alpha \mu \eta \lambda l_{l} \alpha$, celle du père pour sa fille avant le mariage, celle du mari pour sa femme après le mariage. - Sur la liaison entre Aphrodite et les Grâces d'un point de vue politique, ct. J. H. Olivier, op. cit., p. 106-117.

33. Paus., I, 43, 6. 
l'Iliade ${ }^{34}$. Tous les acolytes traditionnels de l'Aphrodite qui préside aux sentiments amoureux sont réunis et l'épiclèse de la déesse, qui évoque l'accomplissement d'une action, le succès d'une entreprise ${ }^{35}$, résonne d'accents similaires ${ }^{36}$. L'origine du culte est impossible à préciser. Si l'on s'en tient aux certitudes offertes par la datation des statues, on peut considérer le Ive siècle comme terminus ante quem, mais il est difficile d'affirmer que la vénération des compagnons d'Aphrodite sur l'agora mégarienne a précédé la réalisation de leurs statues par les maitres sculpteurs.

Il existe un indice permettant d'aller plus loin dans l'interprétation du culte. En effet, un passage de Xénophon parle d'une visite qu'Agésilas roi de Sparte fit à Mégare pendant sa retraite de Béotie en 378/377 avant notre ère ${ }^{87}$. Avant de rencontrer les magistrats de la cité, Agésilas s'est rendu au sanctuaire d'Aphrodite ${ }^{38}$. Il est indubitable que sa démarche concernait moins les prérogatives amoureuses que politiques de la déesse, ce qui donne à penser que le réseau des significations du sanctuaire de l'agora était plus large que ne le laisse apparaitre la confrontation du nom de ses occupants ${ }^{39}$.

\section{ARGOS}

L'agora de la cité, qui comprend bon nombre de tombeaux énumérés par Pausanias, accueille également une statue d'Ar-

34. Gf. P. Weizsäcker, art. cité, col. 1798.

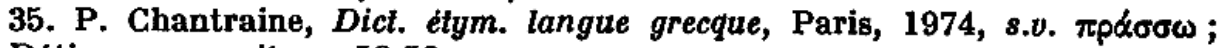
M. Détienne, op. cit., p. 58-59.

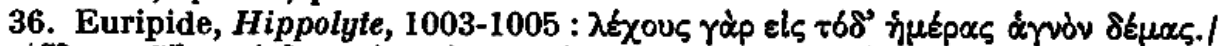

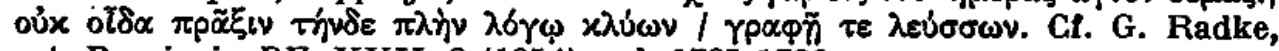
art. Praxis, in RE, XXII, 2 (1954), col. 1785-1786.

37. Xénophon, Hell., V, 4, 58. Cf. R. Legon, Megara. The Political History of a Greek-City State to 336 BC, London, 1981, p. 271-272.

38. Il existe deux sanctuaires de la déesse à Mégare : celui de l'agora et un autre sur l'acropole Karia. La construction du texte de Xénophon implique qu'il s'agit de l'Aphrodite Praxis de l'agora, comme je le montrerai plus en détail dans une étude globale sur les cultes de la déesse en Grèce.

39. Au rr siècle avant $J .-C$., cinq damiurges de la cité ont fait une dédicace à la déesse : $I G$, VII, 41. 
témis Peitho érigée par Hypermnestre - la seule des filles de Danaos à avoir épargné son époux - après qu'elle eut remporté le procès intenté contre elle par son père ${ }^{40}$. Or la tradition locale connaissait une autre fondation de la Danaïde à la suite de son acquittement par le tribunal des Argiens : elle aurait élevé un xoanon à Aphrodite Nikèphoros ${ }^{41}$ que Pausanias a vu dans le sanctuaire d'Apollon Lykios, aux côtés d'Hermès. L'ensemble mythique formé par les mésaventures des Danaïdes et les errances de leur ancêtre, la prêtresse Io, transformée en vache par la colère d'Héra, constitue un " vaste discours sur le mariage en tant que fondement de l'ordre civilisé $~^{\mathbf{4 2}}$ dont les Suppliantes d'Eschyle, et ce que l'on peut deviner des tragédies perdues de la trilogie, offrent la plus belle illustration.

Farouchement opposées au mariage avec les fils d'Egyptos, leurs cousins, les cinquante filles de Danaos arrivent en suppliantes à Argos, pour se mettre sous la protection du roi Pélasgos. A deux reprises, Peitho apparaft : une première fois lorsque le roi entend convaincre son peuple d'accueillir les Supplianles (v. 523) ${ }^{43}$, et une seconde fois, lorsque le chœur des suivantes, conscientes de la démesure de leurs maitresses dans leur refus du mariage et de la violence des prétendants $^{44}$, invoque Cypris et ses enfants, Peitho et Pothos,

40. Paus., II, 21, 1.

41. Paus., II, 19, 6. - La déesse permet à Hypermnestre de triompher, d'où l'épithète de $N i k e$-phoros. La victoire dont il est ici question n'a donc rien à voir avec la guerre. Sur les aspects guerriers d'Aphrodite, cf. V. Pirenne-Delforge, Les cultes d'Aphrodite en Grèce continentale, Université de Liège [thèse dactyl.], 1992 , p. 425-428.

42. F. de Polignac, La naissance de la cité grecque. Cultes, espace et société

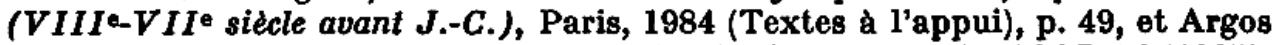
entre centre et périphérie : l'espace cultuel de la cité grecque, in $A S S R, 59$ (1985), p. 55-63. L'auteur insiste beaucoup sur le rôle d'Héra comme protectrice du mariage dans ce contexte mythique. Or, si l'on considère que la tradition locale sur les Danaídes intègre les statues d'Artémis et d'Aphrodite, on peut s'interroger sur la réalité de cette mainmise exclusive.

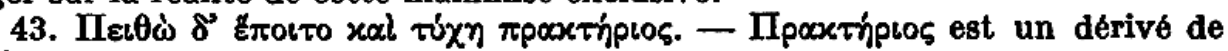

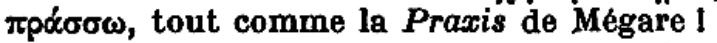

44. L'attribution des vers 1034-1052 aux suivantes n'est que postulée. H. Friis Johansen et E. W. Whittle (Aeschylus : the Suppliants, Copenhagen, 1980, cités par R. Seaford, The Tragic Wedding, in JHS, 107 [1987], p. 114) ont voulu démontrer qu'il s'agissait en fait des gardes du corps argiens. Cela ne change pas grand-chose pour notre propos. 
Persuasion et Désir (v. 1039-1041). A nouveau, persuasion politique et persuasion amoureuse s'épaulent pour fonder l'harmonie au sein du couple et dans la gestion des affaires publiques ${ }^{45}$.

Dès lors, les deux fondations attribuées à Hypermnestre dans l'Argos historique reflètent bien ce que la tragédie donne à voir. C'est en tant que déesses protectrices du mariage, à des titres différents, qu'Aphrodite et Artémis sont invoquées : Artémis en amont et Aphrodite ${ }^{46}$ en aval. La persuasion qui qualifie Artémis dans ce cas signifie bien que la déesse n'est pas ici la vierge qu'invoquent les ennemies du mariage et de la sexualité, mais la protectrice du passage de l'état de jeune fille à celui de femme accomplie ${ }^{47}$.

Allons plus loin. Une tradition ${ }^{48}$ veut que Peitho ait été l'épouse de Phoronée ${ }^{49}$, que Pausanias désigne comme le premier homme à avoir habité l'Argolide, l'arbitre de la querelle entre Héra et Poséidon pour la possession du territoire, l'initiateur d'un synécisme primitif ${ }^{50}$ et l'inventeur du feu en lieu et place de Prométhée ${ }^{51}$. Cette énumération souligne à

45. Cf. Froma I. Zeitlin, La politique d'Eros. Féminin et masculin dans les Suppliantes d'Eschyle, in Metis, 3 (1988), p. 234, 242-246, 253-255.

46. La présence d'un xoanon d'Hermès à ses côtés n'est peut-être pas indifférente. Sur le rôle d'Hermès dans le mariage, cf. L. Kahn, op. cit., p. 180.

47. Cf. par ex. : C. Calame, Les chceurs de leunes filles dans la Grece archatque. I : Morphologie, fonction religieuse et sociale, Rome, 1977, p. 174-190, 250 (cependant les attributions des divinités qui patronnent les différentes phases de la vie de la femme ne sont pas aussi tranchées que l'auteur le laisse entendre. Il est plus prudent aux p. 222-223) et p. $417:$ Artémis peut contribuer \& à l'intêgration de l'adolescente à la sexualité adulte »; P. Brulé, op. cit., p. 240 et passim. - Le superbe fragment 44 Nauck $^{2}$ des Danaldes, troisième morceau de la trilogie, exalte les pouvoirs d'Aphrodite sur la fertilité et la fécondité. On a supposé - avec une très grande vraisemblance - que c'est le choix d'Hypermnestre d'épargner Lyncée qui était ici approuvé : l'union fondée sur l'amour et la persuasion a triomphé ; les mariages fondés sur la force ont tourné au désastre. Il est très vraisemblable que la comédie satyrique Amymonè connaissait une répétition du thème dans un autre registre. Cf. R. P. WinningtonIngram, The Danaid Trilogy of Aeschylus, in JHS, 81 (1961), p. 141-152; R. G. A. Buxton, op. cit., p. 89-90. - Sur les relations complexes des Danaídes au mariage, cf. R. Seaford, art. cité, p. 110-119.

48. Schol. Euripide, Oreste, 932. 1116.

49. Ou bien la femme d'Argos, l'éponyme de la cité : schol. Euripide, Phén.,

50. Paus., II, 15, 5. Sur Phoronée, cf. Platon, Timée, $22 a$; Apollodore, II, 1, 1 ; Pline, Hist. nat., VII, 193 ; Hygin, Fab., 124, 143, 145, 274.

51. Paus., II, 19, 5. 
suffisance le caractère civilisateur du personnage qui a présidé aux destinées primitives d'Argos ${ }^{52}$. Lui faire épouser la Persuasion $^{53}$ donne un relief supplémentaire à son action et cette association n'est pas sans rappeler l'intervention conjuguée d'Aphrodite et de Peitho au cours du synécisme athénien de Thésée.

\section{SICYONE}

Jusqu'à présent, les témoignages considérés ont fait apparaitre Peitho honorée conjointement avec d'autres divinités. A Sicyone, elle possède un sanctuaire à part entière et le mythe étiologique de sa fondation ne laisse pas d'intriguer. C'est à nouveau Pausanias notre seul témoin ${ }^{54}$.

Après le meurtre de Python, Apollon et Artémis seraient venus à Sicyone, alors appelée Aigialeia, afin de se purifier. Saisis de frayeur en un lieu baptisé Phobos, ils s'en vont en Crète tandis qu'une maladie (nosos) s'abat sur Aigialeia. Les habitants consultent des manteis qui leur enjoignent d'apaiser les dieux en envoyant, en suppliants à la rivière Sythas, sept jeunes garçons et sept jeunes filles. Les enfants parviennent à fléchir les jumeaux divins qui acceptent de se rendre dans la cité. L'endroit où il atteignent l'acropole devient un sanctuaire de Peitho. Depuis lors, pour la fête

52. A Athènes, Cécrops possède maints traits qui l'apparentent à Phoronée : une tradition mythique veut qu'il ait réuni les hommes en une cité (asty), inventé le mariage et arbitré la querelle pour la possession de l'Attique survenue entre Athéna et Poséidon. Cf. Nicole Loraux, Les enfants d'Athéna. Idées athéniennes sur la citoyenneté et la division des sexes, Paris, $1984^{2}$, p. 30.

53. Pausanias (II, 21, 1) se fait l'écho d'une tradition divergente : il a vu sur l'agora d'Argos le tombeau de la femme de Phoronée qui porte le nom de Kerdo - l'astucieuse, la rusée ", tandis qu'une scholie à Platon, Timée, 22, l'appelle Télodikè (id. chez Apollodore, II, 1, 1, 3; Tzétzes, schol. à Lycophron, Alexandra, 177) : le champ sémantique évoqué par ces noms insiste chaque fois sur le caractère civilisateur de Phoronée et met en évidence les moyens de son action. Le fait que Kerdo évoque la ruse permet peut-être de supposer que la Peitho est ici puissance ambiguë. Sur ce point, cf. M. Détienne, op. cit., p. 65-66 et n. 101.

54. Paus., II, 7, 7-8. 
d'Apollon, les enfants s'en vont au bord du fleuve Sythas, puis conduisent les "dieux" - sans doute des statues - dans le sanctuaire de Peitho et les ramènent enfin dans le temple d'Apollon.

On a depuis longtemps reconnu la signification initiatique de ce rituel ${ }^{55}$, dont Angelo Brelich a minutieusement analysé les diverses composantes. Nous retiendrons sa conclusion. Le rite d'initiation juvénile originel aurait été inséré, à une époque impossible à déterminer, dans un ensemble religieux où, semble-t-il, Artémis avait, comme ailleurs dans des complexes initiatiques de ce type, un rôle prédominant et qui aurait subi une "apollinisation " ou une "delphicisation " au cours d'un processus dont l'apogée, à Sicyone, se situe sous la tyrannie de Clisthène $e^{56}$.

Mais que vient faire Peitho dans cet ensemble rituel ? Brelich, le seul semble-t-il à s'être posé la question ${ }^{57}$, trouve à Argos, dans les traditions que nous avons déjà évoquées, une solution au problème : la liaison entre Artémis et la Persuasion à Sicyone serait du même type qu'à Argos où Peitho a une fonction statale-politico, la proximité des deux cités permettant d'étayer cette possibilità di confluenza. L'hypothèse est plausible, mais il semble possible d'aller plus loin, en fonction, précisément, des caractères généraux de la

55. M. P. Nilsson, Griechische Feste von religioser Bedeutung, Leipzig, 1906, p. 171-172 ; A. Brelich, Paides e parthenoi, Rome, 1969 (Incunabula Graeca, 36), p. 378-387; C. Calame, op. cit., p. 204-205 (mauvaise lecture du témoignage de Pausanias : le temple d'Apollon ne se trouvait pas au bord du Sythas, mais bien sur l'agora hellénistique - c'est-à-dire sur l'ancienne acropole) ; F. de Polignac, op. cit., p. 77 (mauvaise lecture de Pausanias); P. Brulé, op. cit., p. 219.

56. A. Brelich, op. cit., p. 386-387. De surcroit, Sicyone semble avoir connu une tradition locale du meurtre de Python: Hésychios, s.v. Tołlou Bpouvóc. - J. Fontenrose (Python. A Study of Delphic Myth and its Origins, Univ. of California Press, $1980^{2}$ [1959], p. 57, 86-87, 384) ne semble pas considérer qu'il ait pu s'agir d'un rite d'initiation; il ne prend en compte que la légende qui retrace le meurtre de Python. Quant à la présence d'Artémis, elle serait * clariflée par des mythes orientaux * (p. 87, n. 3), proposition à laquelle on ne pourra souscrire. Signalons que les propos de l'auteur à la page 384 sont en complète divergence avec le texte de Pausanias sur lequel ils sont censés se fonder.

57. Ibid., p. 382-383. 
Peitho que nous avons dégagés pour d'autres cités. Le déplacement des enfants jusqu'au Sythas qui, précisons-le, se situait à quelque $15 \mathrm{~km}$ du centre urbain, était une sorte de retraite aux marges de la cité ${ }^{58}$, telle qu'on en trouve ailleurs, avant l'agrégation des jeunes comme membres à part entière de la société dont ils assureront la continuité ${ }^{59}$. Les jumeaux divins assument fréquemment, dans ce type de contexte, le rôle de courotrophes, Artémis protégeant plus particulièrement les filles et Apollon les garçons ${ }^{60}$ pendant la période critique où l'enfant n'en est plus tout à fait un sans être encore un adulte. Peut-être le rite initial comprenait-il une épreuve probatoire dont le mythe du meurtre de Python aurait conservé un souvenir déformé. En effet, il est probable que l'endroit appelé Phobos dans le mythe se situait près du Sythas, là où devaient se rendre les garçons et les parthenoi. La " terreur" caractérise dès lors la période transitoire hors de la cité, en un lieu qui signifie l'opposition à la vie civilisée. Après leur contact avec le monde des marges, les enfants reviennent dans la cité, dans le sanctuaire de Peitho61. Il est clair que Peitho symbolise ici les valeurs typiques de la cité qui s'opposent ainsi à leur contraire, incarné par Phobos. Or, si l'on se rappelle combien les attributions cultuelles de Peitho mêlent étroitement la vie en société et les relations amoureuses, on peut penser que c'est également la divinité qui patronne l'harmonie conjugale, hors de toute violence, dans le temple de

58. Le Sythas marque la frontière entre la Sicyonie et l'Achaie : Paus., VII, 27, 12 (avec une conjecture très plausible). Il s'agit de l'actuel fleuve Tpux

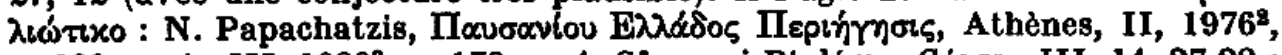
p. 100 , n. 4 ; III, $1980^{2}$, p. 173 , n. 4. Cl. aussi Ptolém., Géogr., III, 14, 27-28 :

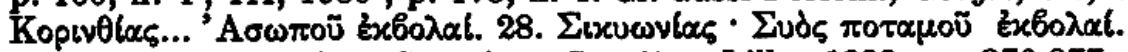

59. H. Jeanmaire, Couroi et Couretes, Lille, 1939, p. 276-277, 285-286 ; P. Brulé, op. cit., p. 400. Cf. aussi M. Massenzio, La festa di Artemis Triklaria e Dionisio Aisymnetes, in SMSR, 39 (1968), p. 100-132.

60. Cf. C. Calame, op. cit., p. 205-206. - Il faut cependant souligner qu'Artémis patronne en certains lieux l'intégration des éphèbes (par ex. Artémis Orthia à Sparte).

61. Il est significatif que, même si les enfants a persuadent , les dieux au bord du Sythas, c'est dans la cité qu'est fondé le sanctuaire de Peitho. F. Bolte, art. Sythas, in $R E$, IV A 2 (1932), col. 1837, situe erronément le sanctuaire de Peitho au bord du Sythas. 
laquelle les enfants amènent les statues d'Artémis et d'Apollon ${ }^{62}$.

Avant de terminer cette visite à Sicyone, revenons un instant sur le témoignage de la Théogonie d'Hésiode. Parlant de la descendance d'Okéanos et de Téthys, il cite d'abord les fleuves tourbillonnants, puis il écrit : «Elle [Théthys] enfantait aussi une race sainte de filles qui, avec sire Apollon et les Fleuves, nourrissent la jeunesse des hommes et tiennent ce lot de Zeus même. "13 Commence alors la liste des noms qui compte Peitho en première position. Tout comme Peitho apporte son aide dans la formation de Pandore, elle semble participer à la formation de kouroi et de korai mortels ${ }^{64}$. Et le culte de Sicyone vient peut-être, si notre analyse est exacte, illustrer cette attribution particulière ${ }^{65}$.

62. I faut cependant préciser qu'aucun élément ne nous permet de dater l'intervention de Peitho dans ce rituel d'agrégation de la jeunesse. L'opposition peitho/phobos n'a vraisemblablement pas été conceptualisée dès les origines de la cité comme l'ouvrage de F. de Polignac (op. cit., p. 77) tend à le laisser penser. - Je tiens à remercier ici Pierre Bonnechère de l'Université catholique de Louvain pour ses remarques judicieuses concernant les rites d'initiation.

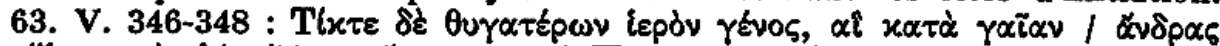

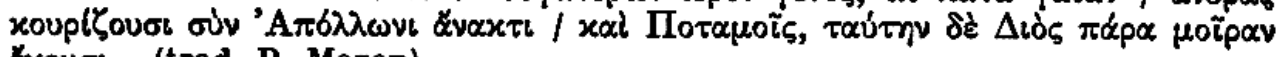
Exouch... (trad. P. Mazon). 265.

64. Cf. les excellentes remarques de M. L. West, Hesiod Theogony, p. 263-

65. On a conservé, parmi les inscriptions des sièges du théâtre de Dionysos à Athènes, la mention d'une prêtesse de Déméter Kourotrophos et Peitho (IG, $\left.\mathrm{II}^{2}, 5131\right)$. Or on connaft, grâce à Pausanias $(I, 22,3)$, un sanctuaire de Déméter Ghloé et de Gê Kourotrophos non loin de celui d'Aphrodite Pandémos et Peitho. Ce genre d'inscription, datant du $\mathrm{II}^{\circ}$ siècle de notre ère, est difficile à interpréter, mais on peut supposer que, à l'époque romaine, les deux sanctuaires étaient desservis par la même prêtresse. Une autre inscription, de provenance et de date identiques $\left(I G, I^{2}, 5149\right)$, unit dans les attributions d'une prêtresse Aphrodite Pandémos, la Nymphe, et une troisième divinité au nom impossible à lire. La Nymphe en question doit être la * propriétaire * du sanctuaire découvert en contrebas de l'Odéon d'Hérode Atticus, sanctuaire détruit au début du I ${ }^{\text {er }}$ siècle avant J.-C. On peut concevoir que son culte ait été transféré au flanc sud-est de l'Acropole, auprès des divinités téminines qui s'y trouvaient rassemblées. Etant donné l'intervention incontestable de la Nymphe dans le cadre des mariages (on a trouvé une très grande quantité de loutrophores dans les fouilles; malheureusement tout ce matériel n'a pas encore fait l'objet d'une publication), le choix de son déplacement dans le sanctuaire d'Aphrodite et de Peitho, dont la desservante s'occupait peut-être aussi de Ge Kourotrophos et de Déméter Chloé (l'inscription modifle cependant l'attribution de l'épithète), permet de préciser la signification de ces cultes, intimement lies au cours de la vie humaine. Cf. Al. N. Oikonomides, The Two Agoras in Ancient Athens. A New Commentary on Their History and Development. Topography and Monuments, Chicago, 1964, p. 8-9. 
CONGLUSION

Dans le jeu des oppositions entre sauvagerie et civilisation, animalité et humanité, injustice et justice, barbarie et grécité, peitho se place du côté positif, en regard de la violence ${ }^{66}$. Les textes ${ }^{67}$ nous ont conservé maintes illustrations de cette mise en perspective des valeurs dont l'étude permet une prise de conscience de la manière dont la société grecque se perçoit et se donne à voir. Une lecture des cultes de Peitho permet de retrouver, inscrit dans la vie religieuse de la polis, un autre type d'illustration de ces valeurs, preuve qu'il est difficile, et même peu souhaitable, de dissocier la peitho " profane " de la peitho " religieuse ".

La persuasion se place en antithèse des rapports fondés sur la domination arbitraire et violente, que ce soit entre un chef et ses sujets ou entre un homme et une femme, les deux domaines étant intimement liés ; en effet, c'est en tant que structure de base d'une société que l'institution matrimoniale se doit d'être la plus harmonieuse possible, car elle permet aux humains de dépasser la promiscuité sexuelle violente et indifférenciée des origines ${ }^{68}$. D'avant la cité donc.

66. Cf. R. G. A. Buxton, op. cit., p. 58-63.

67. Par ex., Isocrate, Nicoclè (III), 5-9 = Sur l'échange (XV), 253-257; Lysias, Oraison funebre, 18-19. Sur Platon, cf. A. Motte, Persuasion et violence chez Platon, in $A C, 50$ (1981), p. 562-577.

68. Il faut souligner que la peitho peut revêtir d'autres atours que la sociabilité et l'harmonie conjugale; ses rapports avec la ruse ne sont pas à négliger, mais n'interviennent pas dans notre propos. Cf. M. Détienne, op. cit., p. 64-68; L. Kahn, op. cit., p. 131-136 et passim; R. G. A. Buxton, op. cit., p. 63-66. 
Le corpus épigraphique concernant Peitho, dans la mesure où rien ne nous a échappé, comporte une dizaine d'inscriptions $^{70}$.

Thasos offre la mention la plus ancienne ( $v$ siècle av. J.-G.) une borne qui atteste simplement l'existence d'un hieron de Peitho ${ }^{71}$, tandis qu'un règlement de culte thasien $d u{ }_{I^{\prime}}{ }^{e}$ siècle av. J.-G. fait état de l'interdiction de sacrifier une ehèvre ou un porcelet à la déesse ${ }^{72}$. Une loi sacrée de Mytilène (III ${ }^{\mathrm{e}}$ siècle av. J.-C.) publie un interdit semblable, limité au porc, pour un autel d'Aphrodite, de Peitho et d'Hermès ${ }^{73}$. A Paros, un certain Thrasyxénos, connu par une dédicace à Aphrodite Timouchè ${ }^{74}$, en a consacré une autre à Peitho et aux Charites ${ }^{75}$.

A Olynthe, en Macédoine, a été amenée au jour une dédicace (II ${ }^{\mathrm{e}}$ siècle av. J.-G. ?) à Peitho due à trois agoranomes apparemment sortis de charge ${ }^{76}$, tandis qu'un groupe de magistrats religieux rhodiens (archiérothyte, hiéropes, épi-

69. Pour l'iconographie de Peitho, identiflée par une inscription, cf. Erika Simon, art. Peitho, in Enciclopedia dell' arte antica classica e orientale, VI (1965), p. 5-8. Voir aussi A. Shapiro, art. cité, p. 9-22.

70. Pour les deux inscriptions athéniennes, cf. supra, notes 21 et 65.

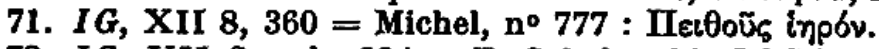

72. IG, XII Suppl., $394=$ F. Sokolowski, $L S C G$, no 73. Cet interdit $8 \theta$ retrouve pour d'autres divinités : $\mathrm{cf}$. Ch. Picard, Un rituel archaique du culte de l'Héraclès thasien trouvé à Thasos, dans $B C H, 47$ (1923), p. 241-274, surtout p. 244-246. Voir aussi $J$. Pouilloux, in Etudes thasiennes, 3 (1954), p. 333-334, 340-341.

73. IG, XII 2, $73=$ Michel, n* 709. Il s'agit peut-être d'Aphrodite Peitho. La même ambiguîté apparaft dans une inscription de Cnide (Iv ${ }^{\theta}-11^{\mathrm{e}}$ siècle av. J.-C.) : SE G, XII, 42 ; cf. Bull. épigr., 67 (1954), no 228 : comme l'inscription est illustrée par un bas-relief représentant deux femmes, L. Robert penche en faveur de la juxtaposition des deux divinités dans la dédicace. - Problème encore pour une inscription de Pharsale : $I G$, IX 2, 236.

74. IG, XII 5, 222.

75. W. Peek, in $M D A I(A), 59$ (1934), p. 60, no 18 (non datée par l'éditeur).

76. D. M. Robinson, A New Inscription from Macedonia, in $A J A, 37(1933)$, p. 602-604. Voir les remarques virulentes de L. Robert, in Opera minora selecta, III (1969), p. 1570-1571. 
mélète des temenoi) associe Peitho et Hermès dans une dédicace datable des environs de l'an 100 avant J.C. ${ }^{77}$

Ainsi l'épigraphie vient-elle confirmer qu'au moins à partir du ve siècle avant notre ère, Peitho recevait un culte et que, même si son association avec Aphrodite est largement attestée, elle était bien honorée comme divinité à part entière. Hermès est l'autre dieu qui l'accompagne. La dévotion des magistrats envers Aphrodite et Hermès est un fait trop connu pour qu'il soit nécessaire d'insister ici ${ }^{78}$; que Peitho se retrouve dans un tel contexte met bien en évidence les nécessités de la concorde et de l'éloquence persuasive qui interviennent dans la charge des magistrats, notamment celle des astynomes, souvent arbitres entre marchands et clients, ouvriers et employeurs.

77. Vassa Kontorini, Rhodiaka I : Inscriptions relatives d l'histoire et aux cultes de Rhodes au $I I^{\circ}$ et au $I^{\circ}$ s. av. J.-C., Louvain-la-Neuve, 1983, p. 67$71=S E G$, XXXIII, n०643.

78. Cf. Bull. épigr., 72 (1959), n $325 ; 77$ (1964), n॰ 82, 288 ; F. Croissant, F. Salviat, Aphrodite gardienne des magistrats : gynéconomes de Thasos et polémarques de Thèbes, in $B C H, 90$ (1960), p. 460-471 ; F. Sokolowski, Aphrodite as Guardian of Greek Magistrates, in $H T h R, 57$ (1964), p. 1-8. 